\title{
Soft physics in ALICE
}

\author{
Angela Badalá *t \\ INFN-Sezione di Catania \\ V. Santa Sofia, 64, Catania (Italy) \\ E-mail: Angela.Badala@ct.infn.it
}

ALICE is the dedicated experiment for relativistic heavy-ion physics at LHC. Following some considerations on the main characteristics of heavy-ion physics expected at the LHC energies, a review of the performance of the ALICE detector in the soft physics domain is discussed. In particular, global event characterization, measurement of identified particle spectra, detection of hadronic short-lived resonances, anisotropic flow determination and HBT correlation studies are discussed.

Correlations and Fluctuations in Relativistic Nuclear Collisions

July 7-9 2006

Florence, Italy

\footnotetext{
* Speaker.

${ }^{\dagger}$ for the ALICE collaboration
} 


\section{Introduction}

ALICE (A Large Ion Collider Experiment) is one of the approved experiments at the CERN Large Hadron Collider. Its main purpose is the study of the strongly interacting nuclear matter and the Quark Gluon Plasma (QGP), the new phase of matter where quarks and gluons are no longer confined inside hadrons and where a partial restoration of the chiral symmetry is expected.

The last two decades have seen a variety of experiments on heavy-ion collisions at ultrarelativistic energies being carried out at CERN and BNL. Extremely interesting results concerning possible evidences of QGP signatures, as the strangeness enhancement and the $\mathrm{J} / \psi$ suppression, were obtained at SPS [1] In recent years, allowing a better understanding of the physics of strongly interacting matter, the RHIC results $[2,3,4,5]$ have confirmed the SPS observations and have added new observables to these phenomena, as the increase of the elliptic flow up to the hydrodynamical limit and the suppression of high- $\mathrm{p}_{t}$ particles (jet quenching).

At LHC, the nucleon-nucleon centre-of-mass energy will exceed that available at RHIC by a factor of about 30, leading to ideal conditions for the formation and study of the QGP. In fact, it is estimated that at LHC the matter in the QGP phase will be hotter, bigger and long-lived with respect to other experiments, since all the parameters of the QGP phase (as energy density, size and lifetime of the system) will be enhanced (see Tab. 1) [6]. The initial temperature will also largely exceed the calculated critical temperature for the QGP formation $\left(\mathrm{T} \sim 3 \mathrm{~T}_{c}\right.$ with $\mathrm{T}_{c} \sim 170$ $\mathrm{MeV}$ [7]). Moreover, it will be possible to study the QCD matter in conditions close to those of the early universe, since the net baryon density in the central region will be essentially vanishing (or zero).

Soft physics concerns the study of hadrons with low $\left(\mathrm{p}_{t}<2 \mathrm{GeV} / \mathrm{c}\right)$ and medium $(2 \mathrm{GeV} / \mathrm{c}<$ $p_{t}<6 \mathrm{GeV} / \mathrm{c}$ ) transverse momentum. A detailed study of the characteristics of these particles and of their correlations is fundamental to understand the evolution of the partonic system formed in the first stages of the collision and the corresponding hadronization processes. Here I will review the performance of the ALICE detector concerning global event characterization, bulk properties determination (by identified particle spectra), detection of hadronic short-lived resonances, expansion dynamics (flow) and space-time measurements by HBT correlations.

The paper is organized as follow. The main characteristics of the physics expected at the LHC energies are discussed in Sect. 2. A short description of the ALICE detector is reported in Sect. 3. The expected performance of the ALICE detector with respect to the above described items are given in the sections from 4 to 8 . More details can be found in the ALICE Physics Performance Report Vol. I and II $[8,9]$.

\section{Heavy-ion physics at LHC}

According to the current schedule, LHC will start its operation at the end of 2007 with p-p collisions at low energy $\left(\sqrt{s}=900 \mathrm{GeV}\right.$ ) and low luminosity (about $10^{27} \mathrm{~cm}^{-2} \mathrm{~s}^{-1}$ ). The first $\mathrm{Pb}-\mathrm{Pb}$ collisions are expected in 2008. Under nominal conditions, it will be possible to study pp collisions at $\sqrt{s}=14 \mathrm{TeV}$ with a luminosity of $10^{34} \mathrm{~cm}^{-2} \mathrm{~s}^{-1}$ and $\mathrm{Pb}-\mathrm{Pb}$ collisions at $\sqrt{s_{N N}}$ equal to $5.5 \mathrm{TeV}$ with a luminosity of $10^{27} \mathrm{~cm}^{-2} \mathrm{~s}^{-1}$. It is expected that each year the LHC beam time will be devoted for several months $\left(10^{7} \mathrm{~s}\right.$ effective time) to $\mathrm{p}$-p data-taking and for a few weeks $\left(10^{6} \mathrm{~s}\right)$ to the study 
Table 1: Comparison of various parameters characterizing heavy-ion central collisions at SPS, RHIC and LHC.

\begin{tabular}{|l|c|c|c|}
\hline \hline Parameter & SPS & RHIC & LHC \\
\hline \hline$\sqrt{s} / A(\mathrm{GeV})$ & 17 & 200 & 5500 \\
Charged particle density $d N_{c h} / d \eta$ & 400 & 650 & $(1.5 \div 6) \times 10^{3}$ \\
Energy density $\left(\mathrm{GeV} / \mathrm{fm}^{3}\right)$ & 2.5 & $4 \div 5$ & $15 \div 40$ \\
Time of initial thermalization $(\mathrm{fm} / \mathrm{c})$ & $\sim 1$ & $\sim 0.5$ & $<0.2$ \\
Volume at freeze-out $\left(\mathrm{fm}^{3}\right)$ & $10^{3}$ & $7 \times 10^{3}$ & $2 \times 10^{4}$ \\
Lifetime of QGP phase $(\mathrm{fm} / \mathrm{c})$ & $<1$ & $2 \div 4$ & $4 \div 10$ \\
\hline \hline
\end{tabular}

of $\mathrm{Pb}-\mathrm{Pb}$ collisions. Moreover, it is also planned to study other systems, as $\mathrm{pA}$ and lighter ions (i.e. $\mathrm{Sn}, \mathrm{Kr}, \mathrm{Ar}, \mathrm{O})$.

The increase of the energy at LHC will open new aspects on the physics which can be probed. While at SPS energies soft collisions were dominant, at LHC hard processes will contribute significantly to the total cross section ( $\sigma_{\text {hard }}$ is estimated to be about $98 \%$ of the total A-A cross-section). Moreover, ALICE will probe a range of low Bjorken- $x\left(10^{-3}-10^{-5}\right)$, accessing a new regime where the initial density of these low- $x$ gluons is expected to be close to saturation of the available phase space. In fact, at sufficiently high density, the gluon fusion becomes competitive with the gluon splitting, limiting the achievable gluon density at small- $x$. As a consequence, particle production is expected at LHC to be determined by the high-density parton distribution, and phenomena like parton saturation and jet quenching should be particularly relevant.

The particle multiplicity reflects the energy density of the produced system. In central $\mathrm{Au}-\mathrm{Au}$ collisions at RHIC energies the events exhibit a charged-particle density at mid-rapidity $\left(\mathrm{dN}_{c h} / \mathrm{d} \eta\right)$ of about 650. Different extrapolations to the LHC energy give for the particle density at midrapidity in central $\mathrm{Pb}-\mathrm{Pb}$ collisions values between 1500 and 3000 [8, 10, 11]. These estimates however are largely uncertain, due to the poor knowledge of different aspects, as the relative importance of soft and hard processes as a function of the energy, the expected presence of a large jet-quenching, which will produce many soft particles, and the effects of structure-function saturation and the nuclear shadowing which may decrease the particle yield.

\section{The ALICE experiment}

In order to explore all the observables needed to characterize the new state of matter, ALICE plans to measure global properties as multiplicity, rapidity distribution, zero-degree energy and bulk properties of the hot and dense medium by identified hadron spectra, hadronic resonances, dilepton continuum and direct photons. The space-time structure and expansion dynamics will be investigated by momentum correlation (HBT), radial and anisotropic flow and fluctuation phenomena. Moreover, also the detection of jets, high $\mathrm{p}_{t}$ particles, open charm and open beauty, which probe the nature of the medium in the early stages, is important. Deconfinement and chiral symmetry restoration will be probed by charmonium and bottonium spectroscopy and by resonance decay, respectively. 
Due to such requirements, the ALICE experiment has to fulfill the following experimental conditions: large acceptance and wide momentum coverage, particle identification of hadrons and leptons, and the accurate measurement of their momentum in a large $\mathrm{p}_{t}$ range. An excellent granularity to cope with the highest expected multiplicity, good vertexes reconstruction, photon and jet detection are also required.

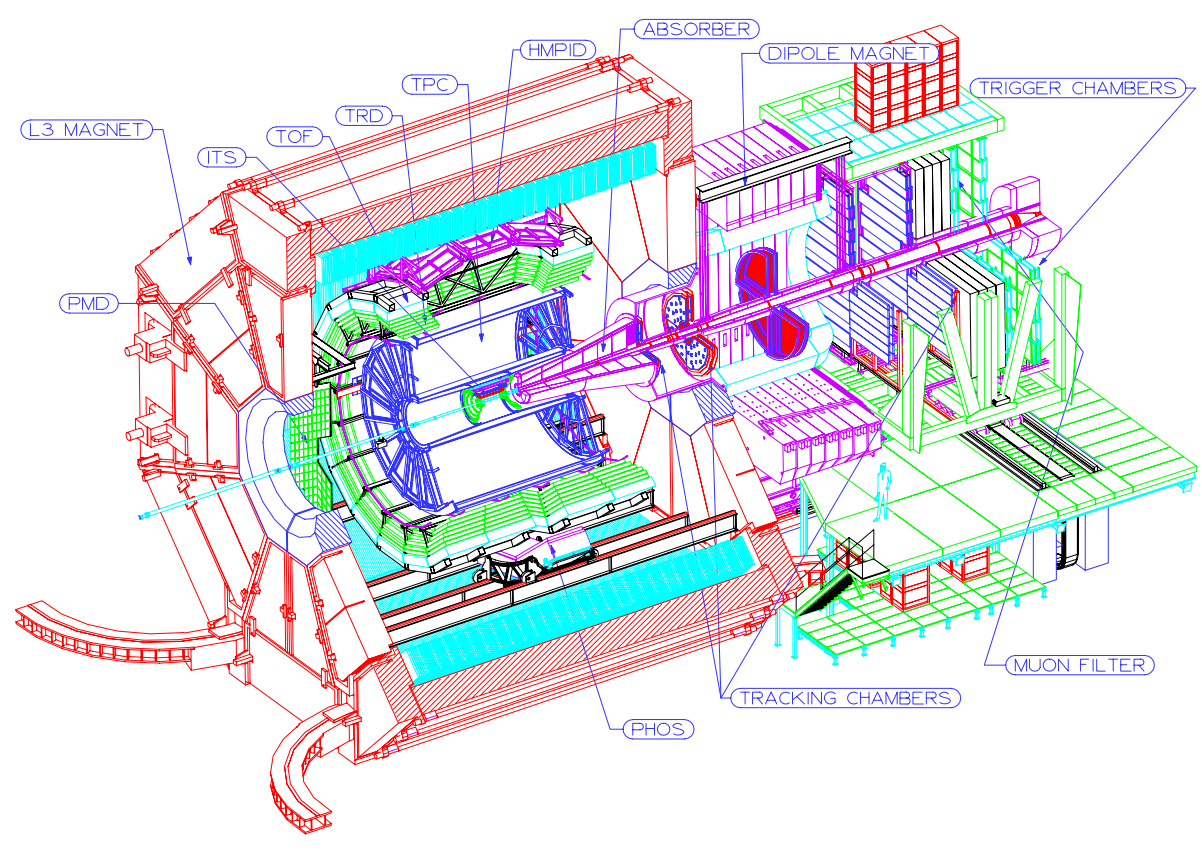

Figure 1: ALICE experimental layout.

The experimental layout is presented in Fig. 1. The ALICE experiment consists of a central detector system, covering the mid-rapidity $(|\eta|<0.9)$ region over the full azimuth, and additional detecting systems placed at forward rapidity. The central system is installed inside the large L3 magnet (15 $\mathrm{m}$ in length and $15 \mathrm{~m}$ in diameter), which generates a weak solenoidal magnetic field $\mathrm{B}$ up to $0.5 \mathrm{~T}$.

The central system includes the central tracking system and the detectors dedicated to the identification of photons and charged particles. The ALICE tracking detectors, designed to cover a broad range of transverse momentum $\left(100 \mathrm{MeV} / \mathrm{c} \leq \mathrm{p}_{t} \leq 100 \mathrm{GeV} / \mathrm{c}\right)$, are: the Inner Tracking System (ITS) $(4 \mathrm{~cm} \leq \mathrm{R} \leq 44 \mathrm{~cm})$, the Time Projection Chamber (TPC) $(90 \mathrm{~cm} \leq \mathrm{R} \leq 250 \mathrm{~cm})$ and the Transition Radiation Detector (TRD) $(2.9 \mathrm{~m} \leq \mathrm{R} \leq 3.7 \mathrm{~m})$.

The ITS consists of six cylindrical layers of coordinate-sensitive silicon detectors. It adopts three different technologies. The two innermost layers are made by silicon pixel detectors, which are fundamental for the determination of the primary vertex as well as for the measurement of the impact parameter of secondary tracks. The Silicon Pixel Detector (SPD) with its cell size of $50 \times 425 \mu \mathrm{m}^{2}$ and its 10 millions channels, will be able to operate with a track density of 80 tracks per $\mathrm{cm}^{2}$ and it will assure a vertexing resolution better than $100 \mu \mathrm{m}$, allowing the reconstruction of charm and beauty particles. Simulations show that a track impact parameter resolution of about $50 \mu \mathrm{m}$ for $1 \mathrm{GeV} / \mathrm{c}$ tracks can be obtained in central $\mathrm{Pb}-\mathrm{Pb}$ collisions.

The middle two and the outer two layers consist of silicon drift detectors and double-sided sili- 
con strip detectors, which allow to identify low momentum particles through $\mathrm{dE} / \mathrm{d} x$ measurements. The ITS in its stand alone mode behaves as a low momentum spectrometer, extending the ALICE tracking ability down to transverse momenta below $100 \mathrm{MeV} / \mathrm{c}$, and improving the momentum resolution.

The main tracking system in ALICE is the TPC. It is the world's largest time-projectionchamber, with a detection volume of $88 \mathrm{~m}^{3}$, filled with a $\mathrm{Ne}(90)-\mathrm{CO}_{2}(10)$ gas mixture. The choice of its inner and outer radii has been dictated by the maximum hit density $\left(0.1 \mathrm{~cm}^{-2}\right)$ and by the track length needed to achieve a $\mathrm{dE} / \mathrm{d} x$ resolution better than $10 \%$. It has two drift volumes of 2.5 $\mathrm{m}$ each, from the middle high-voltage membrane towards the two end-wheels, instrumented with multiwire proportional chambers using the cathode-pad readout $(570,000$ read-out channels). It has been designed to give good performance in tracking efficiency, two-track separation and particle identification also in case of large multiplicity (up to $\mathrm{dN}_{\text {charged }} / \mathrm{d} \eta$ equal to 8000), resulting in 20,000 charged primary and secondary tracks in the TPC acceptance. Tracking efficiency better than $95 \%$ can be achieved, even for low transverse-momentum tracks, down to $200 \mathrm{MeV} / \mathrm{c}$, and at the highest charged particle densities. The momentum and the $\mathrm{dE} / \mathrm{d} x$ resolution are slightly dependent on the event multiplicity, reaching values of about $0.7 \%$ and $6 \%$ respectively, at $\mathrm{p}_{t}=1 \mathrm{GeV} / \mathrm{c}$ and $\mathrm{B}=0.5 \mathrm{~T}$.

The inclusion of the TRD information in the track reconstruction improves the momentum resolution at high $\mathrm{p}_{t}\left(\Delta \mathrm{p}_{t} / \mathrm{p}_{t} \sim 3.5 \%\right.$ for $\mathrm{p}_{t}=100 \mathrm{GeV} / \mathrm{c}$ at $\left.\mathrm{B}=0.5 \mathrm{~T}\right)$. However its main goal is to identify electrons with momenta greater than $1 \mathrm{GeV} / \mathrm{c}$. It consists of six modules in the radial direction. Each module is made by radiator foil stacks followed by a drift region and a cathode-pad read-out chamber. The TRD provides a pion-rejection factor of about 100 for momenta larger than $1 \mathrm{GeV} / \mathrm{c}$, with an electron detection efficiency of $90 \%$.

The Time-Of-Flight (TOF) detector, located in the barrel at radial distances between 3.7 and $4 \mathrm{~m}$, covers the full azimuthal range, and it is based on Multi-gap Resistive Plate Chambers (MRPC) giving an intrinsic time resolution better than 80 ps. It is dedicated to the particle identification in the intermediate momentum range. This detector will provide a $\pi / \mathrm{K}$ and a $\mathrm{K} / \mathrm{p}$ separation better than $3 \sigma$, up to a track momentum of $2.5 \mathrm{GeV} / \mathrm{c}$ and $4 \mathrm{GeV} / \mathrm{c}$, respectively.

The High-Momentum Particle Identification Detector (HMPID) will extend particle identification to higher transverse momenta. With this detector, a separation better than $3 \sigma$ will be achieved for $\pi / \mathrm{K}$ and $\mathrm{K} / \mathrm{p}$ up to $3 \mathrm{GeV} / \mathrm{c}$ and $5 \mathrm{GeV} / \mathrm{c}$, respectively. It covers about $11 \mathrm{~m}^{2}$ at a distance of 5 $\mathrm{m}$ from the interaction point. It is made by proximity-focusing Ring-Imaging Cherenkov (RICH) counters covering the pseudo rapidity range $|\eta|<0.6$ although with a reduced azimuthal coverage.

The PHOton Spectrometer (PHOS), located at $5 \mathrm{~m}$ from the beam axis, is a high-resolution, high-granularity electromagnetic calorimeter, made by about 20,000 $\mathrm{PbWO}_{4}$ (lead-tungstate) crystals. It will detect electromagnetic showers in a limited acceptance domain at central rapidity providing photon and neutral meson $\left(\pi^{0}\right.$ and $\eta$ ) identification through the 2-photons decay channel.

A recent proposal for an Electromagnetic Calorimeter (EMCal), covering the $|\eta|<0.7$ range with an azimuthal coverage of $110^{\circ}$, located in the opposite side of the HMPID detector, has been made in ALICE [12]. This new detector, based on the Shashlik technology, will improve the ALICE capabilities in triggering and measuring high-energy jets and direct photons.

Located on one side of the interaction point, the Forward Muon Spectrometer (FMS) has as 
its main goal the study of the charmonium and bottonium states. It covers the pseudorapidity range $-4<\eta<-2.5$ and consists of a hadronic absorber (located inside the L3 magnet) to absorb hadrons and photons from the interaction vertex, a large dipole magnet (placed at about $7 \mathrm{~m}$ from the interaction point, with a field integral of $3 \mathrm{Tm}$ ) and 5 high-granularity tracking stations to track muons. A passive muon filter (an iron wall about $1 \mathrm{~m}$ thick), followed by four planes of Resistive Plate Chambers ( 2 trigger stations) will be used to trigger on dimuon events.

Finally, the ALICE apparatus is completed by several small detector systems (PMD, FMD, V0, T0 and ZDC) placed at large rapidities, which cover specific tasks.

In the region of pseudo-rapidity range $2.3<\eta<3.5$, located on the opposite side with respect to the muon spectrometer, the Photon Multiplicity Detector (PMD), made by proportional chambers with a passive $\mathrm{Pb}$ converter, will measure the multiplicity and the spatial distribution of photons on an event-by-event basis, addressing the physics related to event-by-event fluctuations. The measurement of the charged-particle multiplicity made by the SPD detector will be extended up to the pseudo-rapidity of 3.4 on the muon-spectrometer side and up to a pseudo-rapidity of 5.1 on the PMD side by the Forward Multiplicity Detector (FMD), which consists of five disks of silicon pad detectors arranged around the beam pipe. The V0 detector has the task to provide a fast measurement of the event multiplicity (to be used for the main interaction trigger) and to locate the interaction vertex. It consists of two arrays of scintillators, assembled as disks, with embedded wavelength shifting fibers located at $90 \mathrm{~cm}$ (muon side) and $350 \mathrm{~cm}$ (PMD side) from the interaction point. The task of the T0 detector, which consists of two arrays of Cherenkov quartz counters, placed near the V0 detector, is to generate the timing signal with precision better than 50 ps.

Finally, at the distance of $116 \mathrm{~m}$ on both sides from the interaction point (IP) inside the LHC tunnel, the Zero Degree Calorimeters (ZDC) will measure the impact parameter of the collision, detecting the spectator nucleons. Such calorimeters are made by an assembly of tantalum or brass with embedded quartz fibers. The brass-ZDC set will detect the proton spectators, which at this distance from the IP will be spatially separated from neutrons by the LHC dipole, while the neutron-spectators will be detected by the tantalum-ZDC set. The informations of these zero degree calorimeters will be improved by a set of two forward electromagnetic calorimeters (ZEM). They will be located at about 7 meters from the IP and will cover the pseudo-rapidity range $4.8 \leq \eta \leq 5.7$. They are designed to measure, event by event, the energy of products emitted at forward rapidity, mainly photons generated from $\pi^{0}$ decay. They are made by an assembly of lead with embedded quartz fibers.

More information on the ALICE apparatus can be found in [8].

\section{Global event characterization}

In heavy ion collisions, the determination of the variables which characterize the geometry of the collision is a crucial prerequisite for the understanding of the dynamics of the collision. The impact parameter $b$ as well as the number of participant nucleons $\left(\mathrm{N}_{\text {part }}\right)$ or the number of nucleonnucleon collisions $\left(\mathrm{N}_{\text {coll }}\right)$ can be extracted in a model-dependent way from either the chargedparticle multiplicity or from the zero-degree energy distribution. 


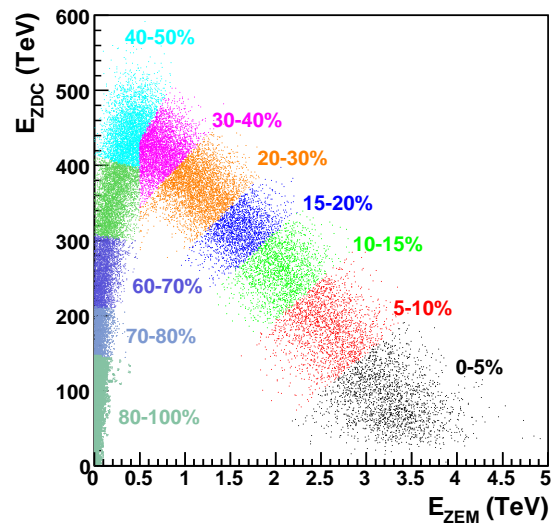

\begin{tabular}{|c|c|c|c|}
\hline$\% \sigma_{\text {TOT }}$ & $<\mathrm{N}_{\text {part }}^{\mathrm{SIM}}>$ & $\left\langle\mathrm{N}_{\text {part }}^{\mathrm{REC}}\right\rangle$ & $\sigma\left(\mathbf{N}_{\text {part }}\right)$ \\
\hline $0-5$ & 386 & 384 & 14 \\
\hline $5-10$ & 329 & 328 & 15 \\
\hline $10-15$ & 276 & 275 & 15 \\
\hline $15-20$ & 231 & 229 & 17 \\
\hline $20-30$ & 177 & 174 & 19 \\
\hline $30-40$ & 120 & 119 & 21 \\
\hline 40-50 & 77 & 77 & 18 \\
\hline $50-60$ & 46 & 48 & 12 \\
\hline $60-70$ & 25 & 24 & 10 \\
\hline $70-80$ & 13 & 12 & 8 \\
\hline
\end{tabular}
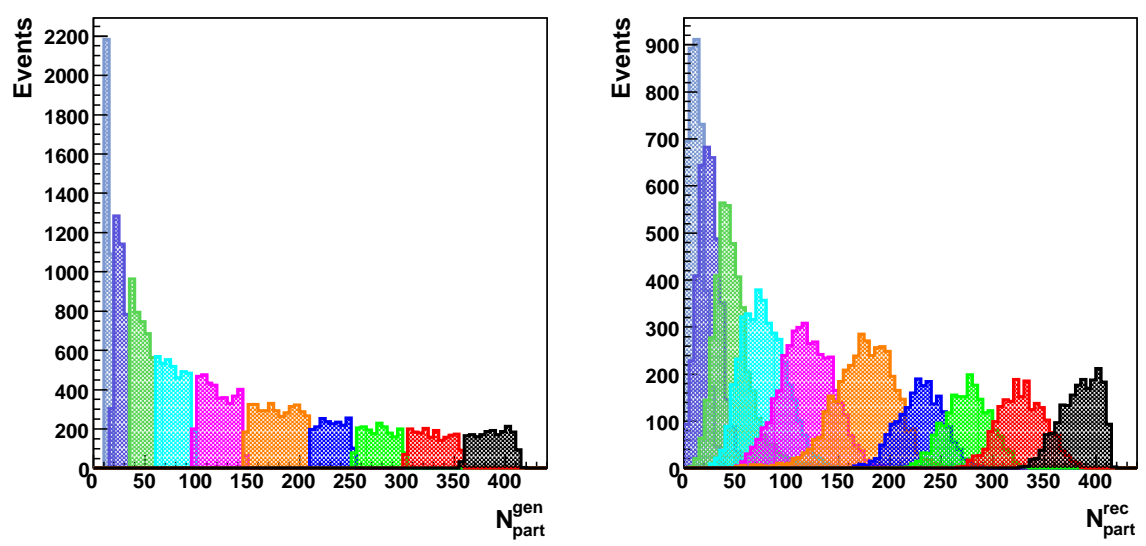

Figure 2: Upper panels: Correlation between the energy deposited in the hadronic ZDCs and in the forward electromagnetic calorimeter. The selected centrality classes are characterized by different colours(left). Table in the right panel: centrality classes defined as a fraction of the total cross section (first column), average value of the generated (second column) and of the reconstructed (third column) number of participants, resolution in the reconstructed $\mathrm{N}_{\text {part }}$ distribution (fourth column). Lower panels: Distribution of the generated(left) and reconstructed (right) number of participants. Different colours are used for the different centrality bins.

\subsection{Centrality determination using the zero-degree calorimeters}

Because of the incomplete fragmentation of the spectator nucleons, there is not a monotonic correlation between the energy measured in the neutron $\left(\mathrm{E}_{Z N}\right)$ and in the proton $\left(\mathrm{E}_{Z P}\right)$ calorimeter and the number of spectator neutrons $\left(\mathrm{N}_{s p e c, n}\right)$ and protons $\left(\mathrm{N}_{s p e c, p}\right)$. For such reason the information given by the ZDC detector must be complemented by additional information originating from other detectors, in order to measure the centrality of the collision. The ZEM permits to solve this ambiguity. In fact, the energy measured by such detector $\left(\mathrm{E}_{Z E M}\right)$, even with a low resolution, increases with the centrality of the collision. This quantity will be then estimated through the correlation between the reconstructed $\mathrm{E}_{Z D C}=\mathrm{E}_{Z P}+\mathrm{E}_{Z N}$ and $\mathrm{E}_{Z E M}$ values. In particular, centrality classes will be defined by means of cuts in the $\mathrm{E}_{Z D C}$ versus $\mathrm{E}_{Z E M}$ correlation (see Fig. 2). In this way it is possible to define centrality classes corresponding to a fraction of the total hadronic cross-section: 


$$
\int_{E_{Z E M}^{i}} \int_{E_{Z D C}^{i}} \frac{d^{2} \sigma}{d E_{Z E M} d E_{Z D C}}=\int_{b_{i}}^{b_{i}+\Delta b_{i}} d b \frac{d \sigma}{d b}=x_{i} \sigma_{t o t}
$$

where the relation between $\mathrm{b}_{i}$ and $\mathrm{x}_{i}$ (fraction of the total inelastic cross section) is determined by the shape of $\mathrm{d} \sigma / \mathrm{db}$ calculated geometrically or by the Glauber model [13]. The corresponding $\mathrm{N}_{\text {part }}$ can be calculated using the Glauber model or the HIJING event generator. There is a good correlation between the generated impact parameter and the reconstructed one. The resolution in the reconstructed impact parameter is of the order of $1 \mathrm{fm}$ over the whole centrality range. In the lower panels of Fig. 2 the distributions of the generated and of the reconstructed number of participants for different centrality bins are reported. The resolution in the calculated $\mathrm{N}_{\text {part }}$, estimated for different fractions of $\sigma_{P b P b}^{i n e l}$, is reported in figure 2(Table). One can note that such quantity is roughly constant for all the centrality bins $\left(\sigma_{N_{p a r t}}=15\right)$ and consequently the relative resolution, $\sigma_{N_{\text {part }}} / N_{\text {part }}$, is about $5 \%$ for central collisions and about $25 \%$ for semi-central collisions ( $\mathrm{b}=8 \mathrm{fm}$ ), values which are comparable to those obtained in heavy-ion experiments at lower energies.

\subsection{Charged multiplicity determination}

The measurement of the charged particle multiplicity is one of the first items of interest in a heavy ion experiment. At ultrarelativistic energies it allows to have important information on the event characteristics, since the distribution of the produced particles is related to the energy density reached in the early phase of the collision. This connection is usually done through the Bjorken formula:

$$
\varepsilon_{B_{j}}=\frac{\left\langle m_{t}\right\rangle}{2 \pi R^{2} \tau_{0}}\left(\frac{d N_{c h}}{d y}\right)_{y=0}
$$

where $\tau_{0}$ is the formation time, $R$ is the initial radius of the expanding system $\left(R=1.2 \mathrm{~A}^{1 / 3} \mathrm{fm}\right)$ and $\left(\mathrm{dN}_{c h} / \mathrm{dy}\right)_{y=0}$ is the charged multiplicity at mid-rapidity.

The multiplicity information permits also to put some constraints on the hadroproduction models, on the importance of hard and soft contribution in the hadron production, and on the role of the high density QCD effects in determining the global event characteristics. Moreover, information on the fragmentation of the projectile and of the target can be extracted from the multiplicity in the forward region.

The ALICE experiment has different detectors, covering different pseudorapidity ranges, which contribute to the multiplicity measurement, and therefore it will use different techniques to reconstruct the multiplicity:

1. counting the clusters in the innermost ITS layer (first SPD layer) $(|\eta|<2)$;

2. counting the tracklets, defined as the association of clusters on the two SPD layers with a straight line to the main vertex $(|\eta|<1.5)$;

3. counting the tracks reconstructed by the full tracking procedure in ITS+TPC $(|\eta|<0.9)$;

4. energy deposition in the pads of the FMD. 

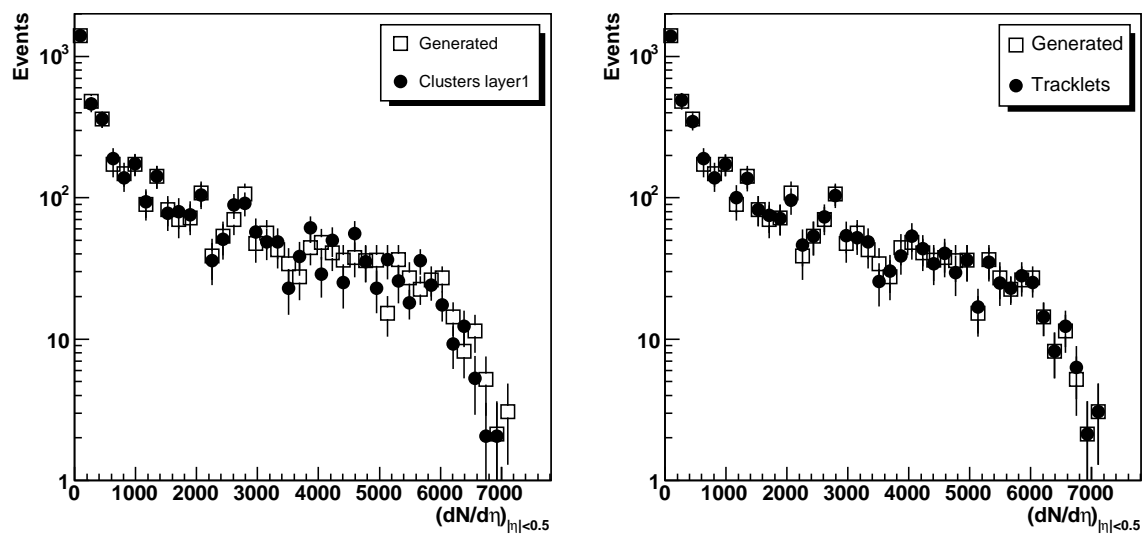

Figure 3: HIJING generated (open symbol) and reconstructed (close symbol) charged multiplicity at midrapidity $(|\eta|<0.5)$. Reconstruction has been done by the clusters counting method (left) and by the tracklets method (right).

In ALICE, thanks to the $\eta$-coverage of the SPD and the FMD detectors the measurement of multiplicity distribution over about $8 \eta$-units will be possible. Information on the performance of the SPD as a multiplicity detector can be found in [14]. Usually a good compatibility between generated and reconstructed charged multiplicity has been found; as an example the comparison between generated and reconstructed charged multiplicity at mid-rapidity $(|\eta|<0.5)$ is shown in Fig. 3 for a set of HIJING events.

\section{Identified particle spectra}

Identified particle spectra provide information about the bulk properties of the collision. In particular, the $\mathrm{p}_{t}$ spectra contain information about the value of temperature at the kinetic freezeout, and on the dynamical evolution of the system, i.e. on the collective flow. Flavour composition allows determination of the chemical freeze-out temperature and chemical potentials.

Data at RHIC and SPS energies are well reproduced by equilibrium statistical models $[15,16$, $17,18]$. In this case it is possible to describe a considerable number of particle ratios with a small number of thermal/statistical parameters such as temperature, volume and chemical potentials. If the systematics observed at lower energies will be preserved at LHC, the expected freeze-out conditions are $\mathrm{T}=(170 \pm 5) \mathrm{MeV}$ and $\mu_{B}=1_{-1}^{+4} \mathrm{MeV}$; i.e. a chemical freeze-out temperature similar to that found at RHIC energies and a vanishing chemical potential.

A prediction for LHC of a non-equilibrium statistical model has become recently available [19]. According to this model, the system is not necessarily in chemical equilibrium and its chemical composition reflects the state and the dynamics of the system in the early phase. The strange-overnon-strange particle ratios in particular vary strongly with the strangeness phase space occupancy $\gamma_{S}$. In the framework of this model, $\gamma_{S}$, which is strictly correlated with the strangeness content in the early phases, is expected to be significantly larger (of the order of $\gamma_{S}=5-10$ ) at LHC than at RHIC or SPS, due to the large density of strangeness at the hadronization following the deconfined phase. Therefore the obtained particle ratios would differ strongly from the equilibrium situation 
$\left(\gamma_{S}=1\right)$, allowing to disentangle between equilibrium and non-equilibrium pictures. At LHC energies the hard processes will play a strong role in the particle production and there will be a large production of jets and minijets; their contribution to the final hadron yield will differ from that originating from an equilibrated system.

SPS and RHIC measurements have shown that particle identification (PID) is crucial for the interpretation of the results. In particular, particle identification is important when measuring quantities as the nuclear modification factor $\left(\mathrm{R}_{c p}\right.$ and $\left.\mathrm{R}_{A A}\right)$, baryon/meson ratio and elliptic flow. Combining the information contained in the measurement of these quantities at RHIC, the results suggest a momentum dependent ordering of the mechanisms which contribute to the particle production. While the region below $4 \mathrm{GeV} / \mathrm{c}$ appears to be dominated by the quark coalescence mechanism, the region above $6 \mathrm{GeV} / \mathrm{c}$ shows the dominance of parton fragmentation [20].

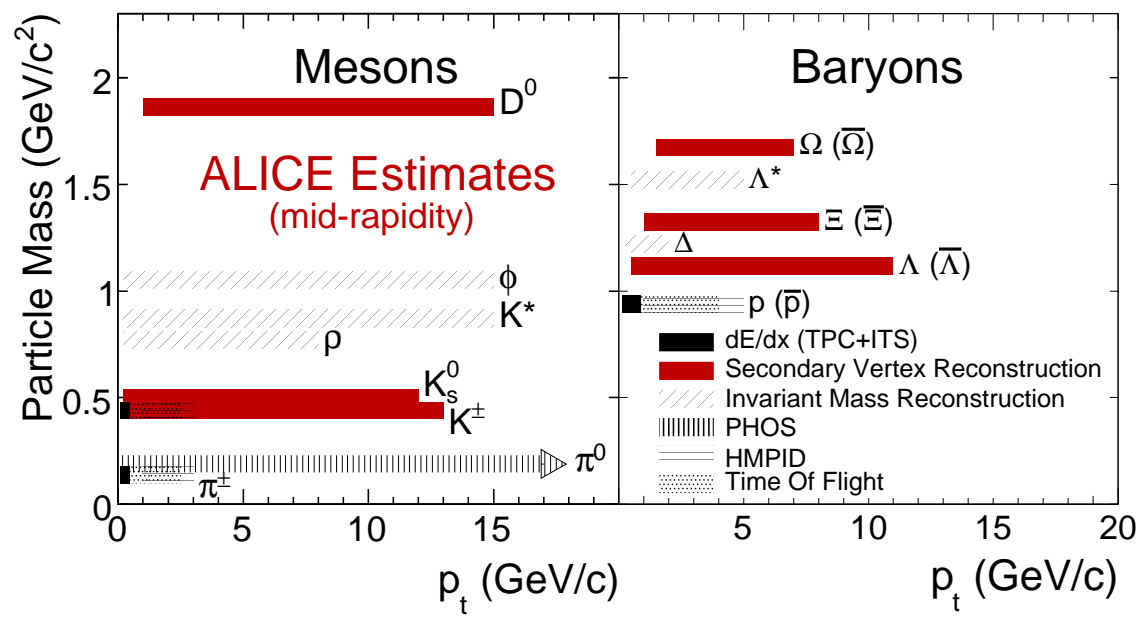

Figure 4: Transverse momentum range for particle identification at mid-rapidity in the ALICE experiment. Mesons and baryons are shown on the left and right panel, respectively. Each range is estimated for 10 millions most central events (i.e. 1-year of data taking). Arrows are drawn when the PID range is larger than $20 \mathrm{GeV} / \mathrm{c}$, i.e. the scale limit in the figure.

The ALICE experiment, combining different detecting systems, described in section 3 , has unique capabilities to reconstruct and identify particles. In Fig. 4 the estimated transverse momentum ranges for identification of particles at mid-rapidity are shown. The estimate has been done for 1-year of data taking (i.e. $10^{7}$ central $\mathrm{Pb}-\mathrm{Pb}$ events). Left and right panels refer to mesons and baryons, respectively. Moreover, using the relativistic rise in the TPC, it would be possible to measure inclusive particle spectra for pions, kaons and protons on a statistical basis and to identify individual tracks of pions and protons with reasonable efficiency and good purity up to momenta of at least $50 \mathrm{GeV} / \mathrm{c}$.

Strange particles can be identified by their weak decay topologies, allowing their identification over a large range of transverse momentum. For these particles the statistical limit in transverse momentum has been estimated by the convolution of spectra (generated with different hypotheses of transverse radial flow) and the reconstruction rate. It has been found that $\Lambda$ 's up to $11-13 \mathrm{GeV} / \mathrm{c}$ and $\Xi$ 's and $\Omega$ 's up to 7-10 GeV/c will be identified [21].

Moreover, due to their high branching ratio in the muonic channel (about 63\%) and to the large 
acceptance of the ALICE central barrel, the identification of $\mathrm{K}^{+}$and $\mathrm{K}^{-}$via their kink topology ( $\mathrm{K}$ $\rightarrow \mu v$ ) seems a promising technique for identifying kaons (event by event) in a momentum range larger than that achieved with the standard ALICE PID.

\section{Short-lived resonance detection}

Short lived resonances (i.e. resonances with a lifetime comparable to that of the collision fireball) may provide information about the later stages in the evolution of the hadronic system, and give an estimate of the time difference between chemical and kinetic freeze-out [22]. In addition, in medium-modifications of their line-shapes are expected, due to interactions of the resonances with the dense medium [24, 25] and as a result of the partial chiral symmetry restoration [23]. Owing to the large rescattering cross section in the medium, the hadronic decay is strongly influenced by final-state interactions. This can result in deviations between the expected thermal yields and those actually measured, as it has been observed in RHIC data [26].

The possibility to reconstruct resonances by their hadronic decay has been largely investigated in ALICE. Studies on the leptonic decay channel are also in progress.
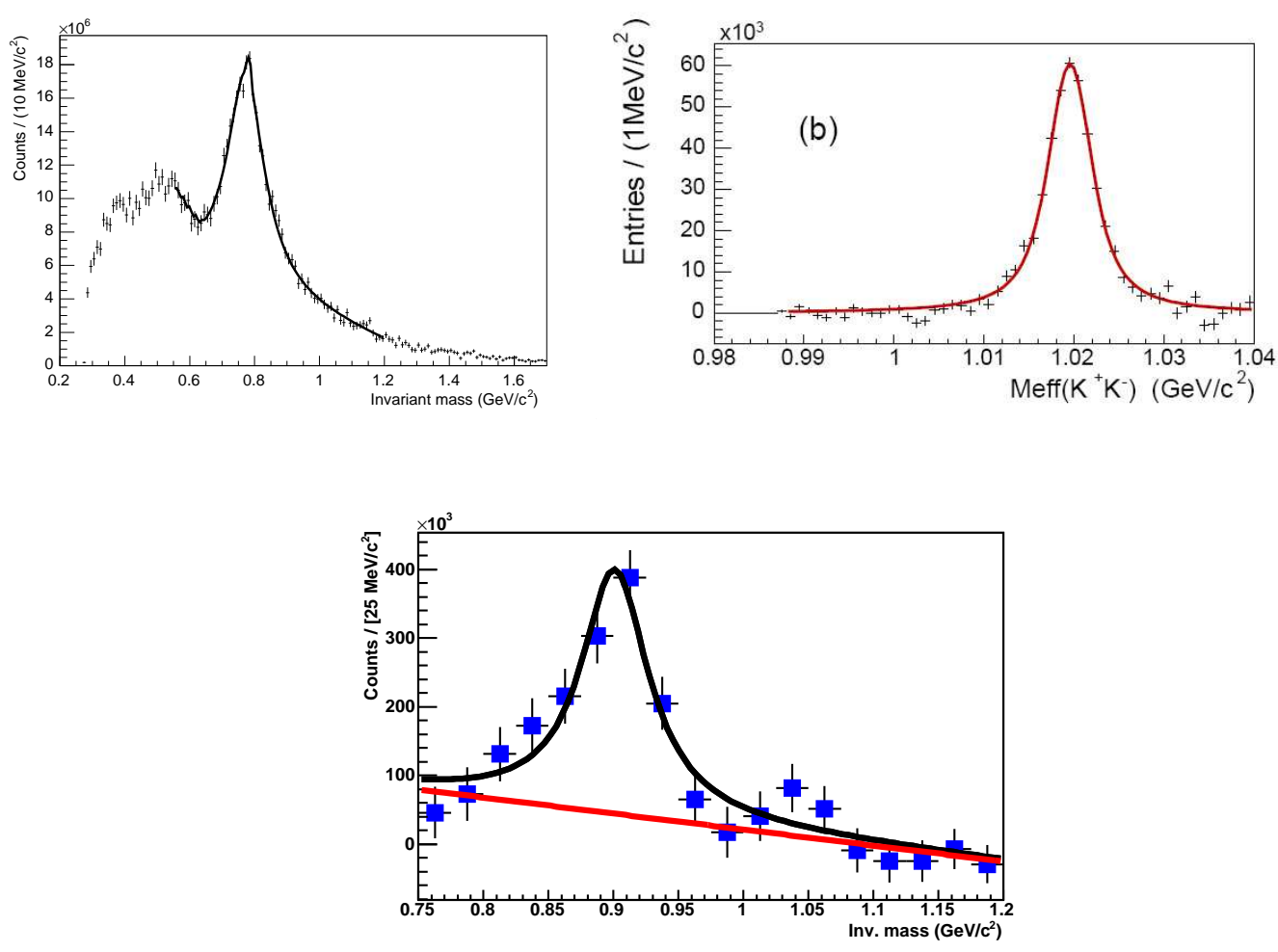

Figure 5: Upper panel: $\rho$ (left) and $\phi$ (right) signal. Lower panel: $K^{*}(892)^{0}+\overline{K^{*}(892)^{0}}$ signal.

Resonances are reconstructed by their invariant mass spectrum. The background is estimated by the like-sign method in $\mathrm{Pb}-\mathrm{Pb}$ events and by the event-mixing technique in $\mathrm{p}-\mathrm{p}$ collisions. The ability of ALICE to detect $\rho^{0}(770), \mathrm{K}^{*}(892)^{0}$ and $\phi(1020)$ resonances has been tested [27, 28, 29]. 
The expected mass resolution is about $2-3 \mathrm{MeV} / \mathrm{c}^{2}$ for the $\rho$ and $\mathrm{K}^{*}$ and about $1.2 \mathrm{MeV} / \mathrm{c}^{2}$ for the $\phi$ resonance. Moreover, it has been estimated that after 1-year of data taking (i.e. $10^{7}$ central $\mathrm{Pb}-\mathrm{Pb}$ events) it will be possible to detect such resonances up to transverse momenta of $8 \mathrm{GeV} / \mathrm{c}$ for the $\rho$ and $15 \mathrm{GeV} / \mathrm{c}$ for $\phi$ and $\mathrm{K}^{*}(892)^{0}$. In Fig. 5(a) and 5(b) the $\pi^{+} \pi^{-}$and $K^{+} K^{-}$invariant mass spectrum after background subtraction ( $\rho$ and $\phi$ signal, respectively) for $10^{6}$ central $\mathrm{Pb}-\mathrm{Pb}$ events are shown. For the $\phi$ spectrum, only pairs with total transverse momentum larger than $2.2 \mathrm{GeV} / \mathrm{c}$ were taken into account. In Fig. 5 (c) the $K \pi$ invariant mass spectrum after background subtraction $\left(K^{*}(892)^{0}+\overline{K^{*}(892)^{0}}\right.$ signal), for about 15000 central HIJING events, is shown. In this case only pairs with total transverse momentum less than $1 \mathrm{GeV} / \mathrm{c}$ were considered.

\section{Anisotropic Flow}

Flow is a collective expansion of bulk matter. In non-central collisions, the initial anisotropy in the transverse configuration space translates into an anisotropy of the transverse momentum distribution of the outgoing particles. An usual way to characterize the anisotropic flow is to use a Fourier expansion of the triple differential invariant distribution.

$$
E \frac{d^{3} N}{d^{3} p}=\frac{1}{2 \pi} \frac{d^{2} N}{p_{t} d p_{t} d y}\left\{1+2 \sum_{n=1}^{\infty} v_{n}\left(p_{t}, y\right) \cos \left[n\left(\phi-\psi_{R}\right)\right]\right\}
$$

where $\phi$ and $\psi_{R}$ are the particle and the reaction-plane azimuth angles in the laboratory frame, respectively. The Fourier coefficients in eq. (7.1) are given by

$$
v_{n}\left(p_{t}, y\right)=\left\langle\cos \left[n\left(\phi-\psi_{R}\right)\right]\right\rangle
$$

where the average is made over all the particles in the event, for a given $\left(\mathrm{p}_{t}, \mathrm{y}\right)$ bin. In this parameterization, the first two coefficients, $v_{1}$ and $v_{2}$, are known as direct and elliptic flow, respectively. The measurement of these quantities is a powerful tool to study the thermodynamic properties of the source. Moreover, the study of higher harmonics of the momentum anisotropy, and in particular their relation with $v_{2}$, should also permit to probe the initial conditions and to disentangle between ideal and viscous fluid [30].

The observed large amount of elliptic flow observed at RHIC provides compelling evidence for strongly interacting matter which, in addition, seems to behave like an almost ideal fluid. In fact, the measured elliptic flow values at RHIC energies are consistent with those expected from ideal hydrodynamics; this has been pointed out as an indication that the created system approaches local thermal equilibrium [4]. However, discrepancies from ideal hydro have been observed in the RHIC data. For example both STAR [31] and PHENIX [32] collaborations reported values of $v_{4} / v_{2}^{2}$ significantly larger (by a factor 2-3) than the ideal-fluid value of $1 / 2$.

Moreover, at RHIC, indications exist of a collective behaviour at pre-hadronic level, which have been suggested from the observation that the measured elliptic flow of mesons and baryons scale with the number of constituent quarks [33].

In Fig. 6(a) theoretical predictions [34] of $\mathrm{v}_{2} / \varepsilon$ versus collision energy using Color Glass Condensate (CGC) estimates for the initial condition are shown. $\varepsilon$ is the spatial eccentricity and is 

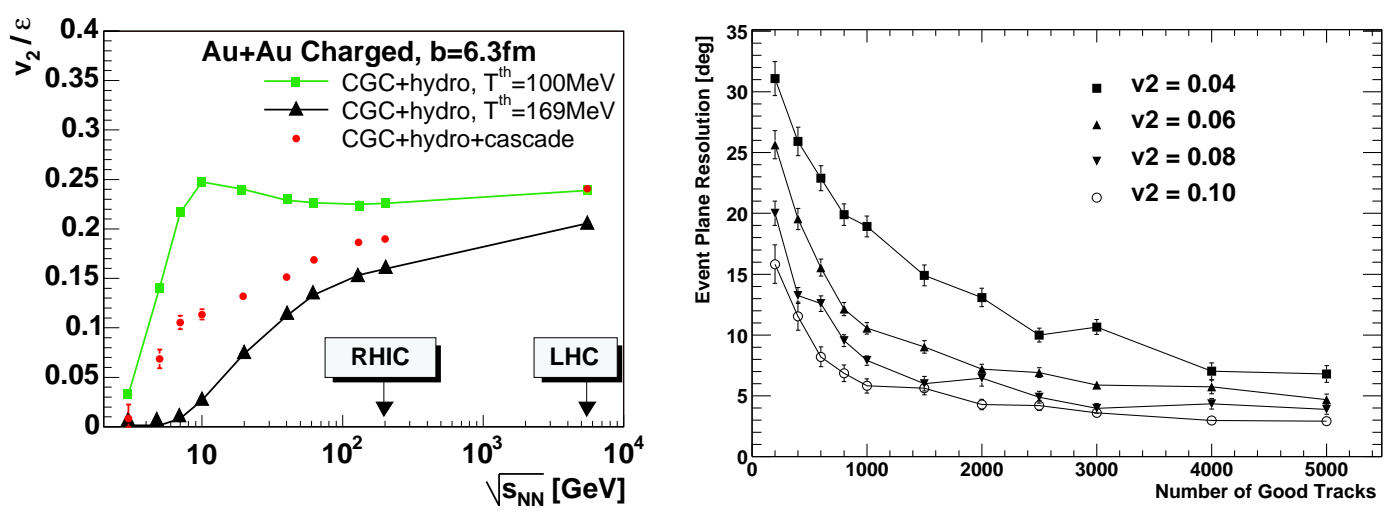

Figure 6: Left panel(a): theoretical prediction of $v_{2} / \varepsilon$ versus collision energy using color glass condensate estimates for the initial conditions [34]. Right panel(b):Dependence of the event plane resolution on multiplicity and elliptic flow.

defined as $\varepsilon=\left\langle y^{2}-x^{2}\right\rangle /\left\langle y^{2}+x^{2}\right\rangle$ where $x$ and $y$ are the spatial coordinates in the plane perpendicular to the collision axis. The brackets denote an average weighted with the initial density. The curve with full squares has been calculated using ideal hydrodynamics up to the kinematic freezeout temperature of $100 \mathrm{MeV}$. The line with triangles represents the contribution to flow from the QGP phase (i.e. hydrodynamics up to $169 \mathrm{MeV}$ ) while the red points represent a more realistic estimate of the flow, i.e. hydrodynamics up to the chemical freeze-out temperature of $169 \mathrm{MeV}$ followed by a hadron cascade description of the final phase. Looking at such results, one can say that at LHC energies the contribution from the QGP phase is much larger than at RHIC or SPS. However these hydro estimates do not take into account the viscosity, which would increase from RHIC to LHC [35, 36] and which could be important in flow estimation [37].

At LHC, $v_{2}$ values of 5-10\% are predicted; as a consequence its measurement should be easy. However, experimental measurements of flow are affected by "non-flow effects", like the azimuthal correlations from jets or mini-jets, whose production is expected to be much larger at LHC than at RHIC energies. First simulation studies in ALICE, have shown that these effects, although present, can be highly reduced by an accurate analysis [38].

To disentangle "non-flow effects" it is important to have independent estimates of the reaction plane and of the elliptic flow from different regions of the phase space. In ALICE these information will come from TPC+ITS, PMD, SPD, FMD and ZDC detectors.

Studies of the reaction plane and the flow determination have been also done in ALICE. For example, in Fig. 6(b) the event plane resolution as a function of the event multiplicity for different flow values is shown. In this case only tracks reconstructed in the TPC were used. One can note that the event plane resolution is better than $8^{0}(\sim 140 \mathrm{mrad})$ as long as the particle multiplicity is larger than 1000 per event and the flow magnitude larger than 0.06 . Furthermore, the reconstructed $v_{2}$ reproduces well the generated transverse momentum dependence. 


\section{HBT correlations}

The study of quantum correlations among hadron pairs makes use of the Hanbury-BrownTwiss (HBT) effect to probe the details of the space-time structure of the source at the decoupling. A standard way to parametrize the source is the Pratt-Bertsch (out-side-long) description, in which the relative momentum vector of the pair $\vec{q}$ is decomposed into a longitudinal direction along the beam axis (long), an outward direction parallel to the pair transverse direction (out) and a sideward direction perpendicular to those two (side). The correlation function is then written as:

$$
C(\vec{q}, \vec{k})=1+\lambda(\vec{k}) e^{-q_{\text {out }}^{2} R_{\text {out }}^{2}-q_{\text {side }}^{2} R_{\text {side }}^{2}-q_{\text {long }}^{2} R_{\text {long }}^{2}}
$$

where the information on the source duration time is put in the out component. Then, the ratio $\mathrm{R}_{\text {out }} / \mathrm{R}_{\text {side }}$ is sensitive to the time duration of the source emission. The hydrodynamical approach at RHIC has been able to describe soft $\mathrm{p}_{t}$ spectra and elliptic flow. However, HBT radii predicted by these calculations are in strong disagreement with the experimental results. In particular, hydrodynamical calculations are not able to reproduce the $\mathrm{p}_{t}$ dependence of $\mathrm{R}_{\text {out }} / \mathrm{R}_{\text {side }}$, which is the same for all the studied systems (pp, dAu and AuAu). This disagreement is known as the "HBT puzzle" $[4,5]$. Some attempts to bring the hydro predictions closer to experimental results pass through an improved treatment of the hadronic stage and/or the introduction of finite viscosity during the hydrodynamical expansion [37]. However, at the moment, this inconsistency between data and models has not been entirely solved.

Obviously, a prediction of the radii expected at LHC is not an easy task. However, a naive prediction can be done considering that the radius depend on the event multiplicity, in particular as $\mathrm{R} \propto\left(d N_{c h} / d \eta\right)^{1 / 3}$. This is seen either from a systematics of RHIC experimental data [39] or from the observation that HBT parameters in central collisions at AGS, SPS and RHIC energies are consistent with a constant mean free path $\left(\lambda_{f} \sim 1\right)$ of pions at freeze-out [40]. Therefore, according to the recent estimates of the multiplicity at LHC, the radius could increase from $15 \%$ up to $60 \%$ respect to the RHIC data.

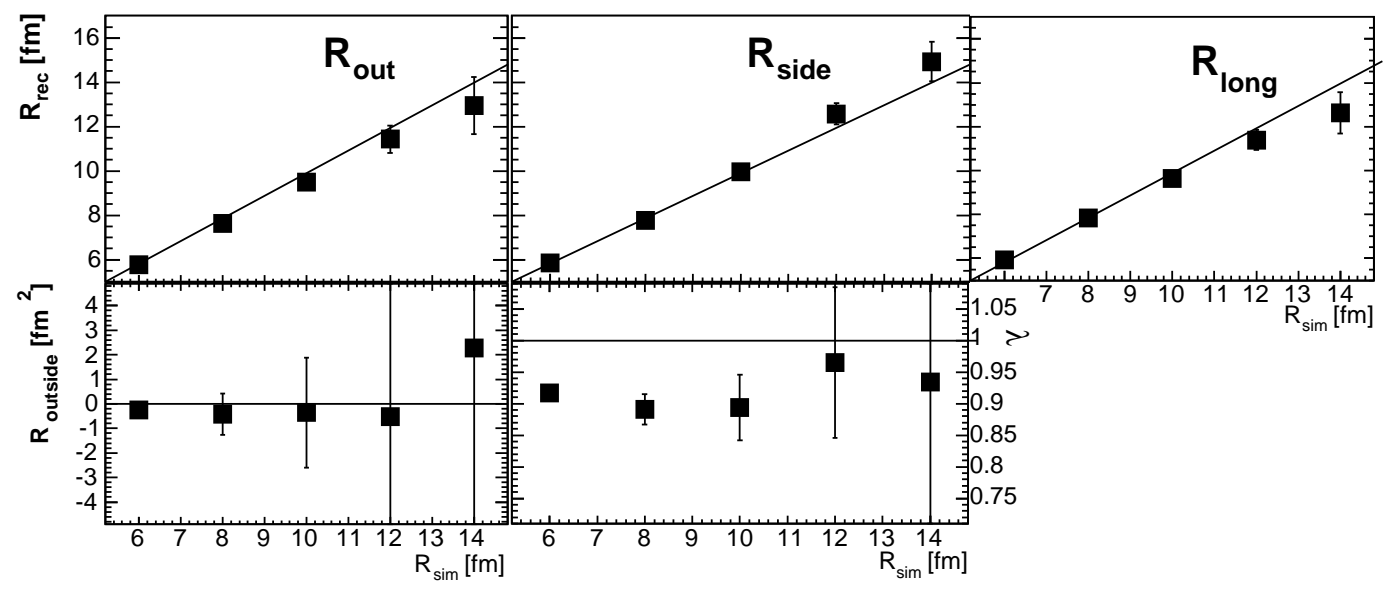

Figure 7: HBT parameters extracted from $\pi^{+} \pi^{+}$pairs with $\mathrm{p}_{t}>500 \mathrm{MeV} / \mathrm{c}$ as a function of the radius of the generated events. 
For a proper treatment of data, studies have been done in ALICE on event mixing and on two track resolutions; track splitting and merging problems have also been investigated. Moreover, methods to take into account the Coulomb interaction have been considered and corrections for momentum resolution and for incorrect particle identification have been calculated. One observes that the shape of the reconstructed correlation function is very close to the generated one and that ALICE is able to reconstruct HBT radii up to $15 \mathrm{fm}$. As an example, the extracted radii compared to the simulated ones, for $\pi^{+} \pi^{+}$with $\mathrm{p}_{t}>500 \mathrm{MeV}$, are shown in Fig. 7 [41].

Due to the high multiplicity expected at LHC energies, it will be also possible to perform an analysis of the correlation functions for single events, which will permit to study source fluctuations on an event-by event basis.

\section{Conclusions}

The construction of the LHC machine and of the ALICE detector are well under way. ALICE is now in a challenging period in which all the detectors have to be completed, tested and placed in their final position in the ALICE cavern. A detailed installation and commissioning plan, endorsed by the LHC Committee, has been established so that ALICE will be complete and ready in time to measure the first pp collisions in November 2007. Many physics working groups are preparing and testing the software tools which will permit to analyze the huge quantity of expected data using the GRID technologies.

LHC will explore new aspects of the strongly-interacting matter. Many of them will be investigated by the so called "soft" probes. The characteristics of the ALICE detector (i.e. particle identification, low $\mathrm{p}_{t}$ cut-off, excellent tracking and vertexing capability) will permit to study the nature of the bulk, its collective behaviour and the influence of the hard processes on its properties in this new energy domain.

\section{References}

[1] U. Heinz and M. Jacob, preprint nucl-th/0002042(2000) and http://info.web.cern.ch/info/Announcements/2000/NewStateMatter/

[2] I. Arsene et al., (BRAHMS Collaboration) Nucl. Phys. A757 (2005), 1.

[3] B.B. Back et al., (PHOBOS Collaboration) Nucl. Phys. A757 (2005), 28.

[4] J. Adams et al., (STAR Collaboration) Nucl. Phys. A757 (2005), 102.

[5] K. Adcox et al., (PHENIX Collaboration) Nucl. Phys. A757 (2005), 184.

[6] K. Safarik, Nucl. Phys. A749 (2005), 229.

[7] F. Karsch and E. Laermann, he-lat/0305025, Quark-Gluon Plasma III, R.Hwa ed.

[8] ALICE Physics Performance Report Vol. I, ALICE Collaboration, J. Phys. G30 (2004), 1517.

[9] ALICE Physics Performance Report Vol. II, ALICE Collaboration, J. Phys. G32 (2006), 1295.

[10] D. Kharzeev, E. Levin and M. Nardi, Nucl. Phys. A747 (2005), 609.

[11] K.J. Eskola et al., Phys. Rev. C72 (2005), 044904. 
[12] ALICE Collaboration, Addendum to the Technical Proposal, Electromagnetic Calorimeter, CERN-LHCC-2006-014 (2006).

[13] C. Oppedisano, ALICE Internal Note 2002-08(2002).

[14] R. Caliandro, R.A. Fini and T. Virgili, ALICE Internal Note 2002-043(2002);

T. Virgili, ALICE Internal Note 2006-013(2006).

[15] A. Andronic, P. Braun-Munzinger and J. Stachel, Nucl. Phys. A772 (2006), 167.

[16] O. Barannikova et al., STAR Collaboration, nucl-ex/0403014.

[17] G. Torrieri and J. Rafelski, hep-p4/0409160, J.Phys. Conf. Ser.5(2005)246.

[18] G. Torrieri, S. Jeon and J. Rafelski, nucl-th/0509067, QM2005 proceedings.

[19] J. Rafelski and J. Letessier, Eur. Phys. J. C45 (2006), 61.

[20] R.J. Fries and B. Muller Eur. Phys. J. C34 (2004), S279.

[21] R. Vernet et al., ALICE Internal Note 2006-011(2006).

[22] G. Torrieri and J. Rafelski, Phys. Lett. B509 (2001), 239; J. Letessier et al., J. Phys.G: Nucl. Part. Phys. 27(2001)427.

[23] R. Rapp and J. Wambach, Adv. Nucl. Phys. 25 (2000), 1

R. Rapp, Phys. Rev. C63 (2001), 054907.

[24] E.V. Shuryak and G.E. Brown, Nucl. Phys. A717 (2003), 322.

[25] R. Rapp, Nucl. Phys. A725 (2003), 254.

[26] J. Adams et al., (STAR Collaboration), Phys. Rev. Lett. 97 (2006), 1323301.

[27] R. Platt and O. Villalobos Baillie, ALICE Internal Note 2005-040(2005).

[28] A. Badalá et al., ALICE Internal Note 2005-039.

[29] A. De Caro et al., ALICE Internal Note 2003-067(2003)

B. Batyunya et al., Particle and Nuclei, Lett. Vol. 2(2005)72.

[30] R.S. Bhalerao et al., Phys. Lett. B627 (2005), 49.

[31] J. Adams et al., (STAR Collaboration), Phys. Rev. C72 (2006), 014904.

[32] H.Masui for the PHENIX Collaboration, Nucl. Phys. A774 (2006), 511.

[33] J. Adamas et al., (STAR Collaboration), Phys. Rev. Lett. 95 (2005), 122301.

[34] T. Hirano, Talk given at the workshop on QGP Thermalization (QGPTH05) Vienna.(http://hep.itp.tuwien.ac.at/qgpth05/)

[35] T. Hirano and M. Gylassy, Nucl. Phys. A769 (2006), 71.

[36] L.P. Csernai J.I. Kapusta and L.D. McLerran, Phys. Rev. Lett. 97 (2006), 152303.

[37] D. Teaney, Phys. Rev. C68 (2003), 034913.

[38] E. Simili, Talk given at Workshop on Soft Physics in Ultrarelativistic Heavy Ion Collisions (SPHIC06), 27-29 September 2006, Catania (Italy). (http://www.ct.infn.it/SPHIC06/)

[39] M.A. Lisa, S. Pratt, R. Soltz and U. Wiedermann, Ann. Rev. Nucl. Part. Sci. 55(2005)357 (nucl-ex/0505014);

M.A. Lisa nucl-ex/0512008.

[40] D. Adamova et al.,(CERES collaboration) Phys. Rev. Lett. 90 (2003), 022301.

[41] P.R. Skowronski et al., ALICE Internal Note 2005-031. 